\title{
PROPER RAINBOW CONNECTION NUMBER OF GRAPHS
}

\author{
Trung DuY Doan ${ }^{1}$ \\ School of Applied Mathematics and Informatics \\ Hanoi University of Science and Technology, Hanoi, Vietnam \\ e-mail: trungdoanduy@gmail.com \\ AND \\ INGO SCHIERMEYER \\ Institut für Diskrete Mathematik und Algebra \\ Technische Universität Bergakademie Freiberg \\ 09596 Freiberg, Germany \\ e-mail: Ingo.Schiermeyer@tu-freiberg.de
}

\begin{abstract}
A path in an edge-coloured graph is called a rainbow path if its edges receive pairwise distinct colours. An edge-coloured graph is said to be rainbow connected if any two distinct vertices of the graph are connected by a rainbow path. The minimum $k$ for which there exists such an edge-colouring is the rainbow connection number $r c(G)$ of $G$. Recently, Bau et al. [Rainbow connectivity in some Cayley graphs, Australas. J. Combin. 71 (2018) 381393] introduced this concept with the additional requirement that the edgecolouring must be proper. The proper rainbow connection number of $G$, denoted by $\operatorname{prc}(G)$, is the minimum number of colours needed in order to make it properly rainbow connected. Obviously, $\operatorname{prc}(G) \geq \max \left\{\operatorname{rc}(G), \chi^{\prime}(G)\right\}$.

In this paper we first prove an improved upper bound $\operatorname{prc}(G) \leq n$ for every connected graph $G$ of order $n \geq 3$. Next we show that the difference $\operatorname{prc}(G)-\max \left\{r c(G), \chi^{\prime}(G)\right\}$ can be arbitrarily large. Finally, we present several sufficient conditions for graph classes satisfying $\operatorname{prc}(G)=\chi^{\prime}(G)$.
\end{abstract}

Keywords: edge-colouring, rainbow connection number, proper rainbow connection number.

2010 Mathematics Subject Classification: 05C15, 05C40, 05 C07.

\footnotetext{
${ }^{1}$ Part of this research was done while the first author was a postdoctoral fellow at the Institute of Mathematics, Vietnam Academy of Science and Technology and he wishes to express gratitude for the very good conditions and the pleasant environments of work there. The first author is also thankful to Prof. Ngo Dac Tan, Prof. Phan Thi Ha Duong, and other people for their help at the time. This research is supported by Vietnam National Foundation for Science and Technology Development (NAFOSTED) under grant number 08/2020/STS01.
} 


\section{REFERENCES}

[1] S. Bau, P. Johnson, E. Jones, K. Kumwenda and R. Matzke, Rainbow connectivity in some Cayley graphs, Australas. J. Combin. 71 (2018) 381-393.

[2] Y. Caro, A. Lev, Y. Roditty, Zs. Tuza and R. Yuster, On rainbow connection, Electron. J. Combin. 15 (2008) \#R57. doi: $10.37236 / 781$

[3] L.S. Chandran, A. Das, D. Rajendraprasad and N.M. Varma, Rainbow connection number and connected dominating sets, J. Graph Theory 71 (2012) 206-218. doi:10.1002/jgt.20643

[4] G. Chartrand, G.L. Johns, K.A. McKeon and P. Zhang, Rainbow connection in graphs, Math. Bohem. 133 (2008) 85-98.

[5] L. Chen, X. Li and Y. Shi, The complexity of determining the rainbow vertexconnection of a graph, Theoret. Comput. Sci. 412 (2011) 4531-4535. doi:10.1016/j.tcs.2011.04.032

[6] D.G. Hoffman and C.A. Rodger, The chromatic index of complete multipartite graphs, J. Graph Theory 16 (1992) 159-163. doi:10.1002/jgt.3190160207

[7] H. Jiang, W. Li, X. Li and C. Magnant, On proper (strong) rainbow connection of graphs, Discuss. Math. Graph Theory 41 (2021) 469-479. doi:10.7151/dmgt.2201

[8] A. Kemnitz, J. Przybyło, I. Schiermeyer and M. Woźniak, Rainbow connection in sparse graphs, Discuss. Math. Graph Theory 33 (2013) 181-192. doi:10.7151/dmgt.1640

[9] A. Kemnitz and I. Schiermeyer, Graphs with rainbow connection number two, Discuss. Math. Graph Theory 31 (2011) 313-320. doi:10.7151/dmgt.1547

[10] D. König, Über Graphen und ihre Anwendung auf Determinantentheorie und Mengenlehre, Math. Ann. 77 (1916) 453-465. doi:10.1007/BF01456961

[11] X. Li and S. Liu, Tight upper bound of the rainbow vertex-connection number for 2-connected graphs, Discrete Appl. Math. 173 (2014) 62-69. doi:10.1016/j.dam.2014.04.002

[12] X. Li, M. Liu, and I. Schiermeyer, Rainbow connection number of dense graphs, Discuss. Math. Graph Theory 33 (2013) 603-611. doi:10.7151/dmgt.1692

[13] X. Li and C. Magnant, Properly colored notions of connectivity - a dynamic survey, Theory Appl. Graphs 0(1) (2015) Art. 2. doi:10.20429/tag.2015.000102

[14] X. Li, Y. Mao and Y. Shi, The strong rainbow vertex-connection of graphs, Util. Math. 93 (2014) 213-223. 
[15] X. Li and Y. Shi, On the rainbow vertex-connection, Discuss. Math. Graph Theory 33 (2013) 307-313.

doi:10.7151/dmgt.1664

[16] X. Li, Y. Shi and Y. Sun, Rainbow connections of graphs: A survey, Graphs Combin. 29 (2013) 1-38.

doi:10.1007/s00373-012-1243-2

[17] X. Li and Y. Sun, Rainbow Connections of Graphs (Springer, New York, 2012). doi:10.1007/978-1-4614-3119-0

[18] I. Schiermeyer, Rainbow connection in graphs with minimum degree three, Combinatorial Algorithms, Lect. Notes Comput. Sci. 5874 (2009) 432-437. doi:10.1007/978-3-642-10217-2_42

[19] V.G. Vizing, On an estimate of the chromatic class of p-graph, Diskret. Analiz. 3 (1964) 25-30, in Russian.

[20] D.B. West, Introduction to Graph Theory (Prentice Hall, 2001).

Received 2 December 2019

Revised 22 April 2020

Accepted 22 April 2020 